wavelengths of light and three different polarization directions. Multiple recording layers may then be combined on a single disk, adding the final spatial dimension, to further increase the storage density. The size and shape of the gold nanorods provide the necessary selectivity for the fivedimensional recording process.

The gold nanorods are patterned through a photothermal reshaping process. This reshaping happens when a nanorod absorbs a laser pulse and heats above its melting temperature, which then causes the nanorod to transform into a spherical particle. The nanorods selectively absorb the laser energy depending on the wavelength and polarization of the light while the threshold for photothermal melting ensures that the writing process is confined within the focal volume of the laser. The aspect ratio and orientation of the nanorods determine the wavelength and polarization sensitivity, respectively. Recordings can be imaged nondestructively using longitudinal surface plasmon resonance mediated by two-photon luminescence.
This nonlinear optical detection mechanism provides much higher angular and wavelength sensitivity compared to linear detection mechanisms used with gold nanoparticles. With the demonstrated ability to record pixel sizes close to the expected diffraction limit and in a manner free from cross-talk, this new optical recording technique has the potential to raise storage density into the terabytes for an optical disk the size of a DVD.

Charles BroOKS

\section{Graphene Nanoribbon Interconnect Resistivity Comparable to Copper}

The unique properties of graphene make the material attractive for a wide range of potential electronic devices. R. Murali, K. Brenner, Y. Yang, T. Beck, and J.D. Meindl at the Georgia Institute of Technology have now experimentally demonstrated the potential for another graphene application: replacing copper for interconnects in future generations of integrated circuits. In the June issue of the Electron Device Letters (DOI: 10.1109/ LED.2009.2020182; p. 611), the researchers provide a detailed analysis of resistivity in graphene nanoribbon interconnects as narrow as $18 \mathrm{~nm}$.

"As you make copper interconnects narrower and narrower, the resistivity increases as the true nanoscale properties of the material become apparent," said Murali, a research engineer in Georgia Tech's Microelectronics Research Center. "Our experimental demonstration of graphene nanowire interconnects on the scale of $20 \mathrm{~nm}$ shows that their performance is comparable to even the most optimistic projections for copper interconnects at that scale. Under real-world conditions, our graphene interconnects probably already out-perform copper at this size scale." Use of graphene for these interconnects could help extend the long run of performance improvements for silicon-based integrated circuit technology.

Beyond resistivity improvement, graphene interconnects would offer higher electron mobility, better thermal conductivity, higher mechanical strength, and reduced capacitance coupling between adjacent wires.

"Resistivity is normally independent of the dimension - a property inherent to the material," Murali said. "But as you get into the nanometer-scale domain, the grain sizes of the copper become important and conductance is affected by scattering at the grain boundaries and at the side walls. These add up to increased resistivity, which nearly doubles as the interconnect sizes shrink to $30 \mathrm{~nm}$."

Experimentally, the researchers began with flakes of multi-layered graphene removed from a graphite block and placed onto an oxidized silicon substrate. They used electron beam lithography to construct four electrode contacts on the graphene, then used lithography to fabricate devices consisting of parallel nanoribbons of widths ranging over 18-52 nm. The three-dimensional resistivity of the nanoribbons on 18 different devices was then measured using standard analytical techniques at room temperature.

The best of the graphene nanoribbons showed conductivity equal to that predicted for copper interconnects of the same width. Because the comparisons were between non-optimized graphene and optimistic estimates for copper, the researchers suggest that performance of the new material will ultimately surpass that of the traditional interconnect material.

Though one of graphene's key properties is reported to be ballistic transport-meaning electrons can flow through it without resistance-the material's actual conductance is limited by factors that include scattering from impurities, line-edge roughness, and from substrate phononsvibrations in the substrate lattice.

Use of graphene interconnects could help facilitate continuing increases in integrated circuit performance once feature sizes drop to approximately $20 \mathrm{~nm}$, which could happen in the next five years, Murali said. At that scale, the increased resistance of copper interconnects could offset performance increases, meaning that without other improvements, higher density would not produce faster integrated circuits.

"This is not a roadblock to achieving scaling from one generation to the next, but it is a roadblock to achieving increased performance," he said. "Dimensional scaling could continue, but because we would be giving up so much in terms of resistivity, we wouldn't get a performance advantage from that. That's the problem we hope to solve by switching to a different materials system for interconnects."

\section{Stream of Sand Behaves like Water}

H.M. Jaeger, J.R. Royer, and colleagues from the University of Chicago have demonstrated that dry granular materials such as sands, seeds, and grains have properties similar to liquid, forming water-like droplets when poured from a given source. The finding could be important to a wide range of industries that use "fluidized" dry particles for oil refining, plastics manufacturing, and pharmaceutical production.

Researchers previously thought dry particles lacked sufficient surface tension to form droplets like ordinary liquids.

"Previous studies of granular streams were able to detect clustering by performing experiments in vacuum and were able to establish that the clustering was not caused by the drag from the ambient air," said Jaeger, a professor in the university's Materials Research Science and Engineering Center. "However, the cause of the clustering remained a mystery." 